\title{
Two-gigahertz repetition-rate, diode-pumped, mode-locked Nd:YLF laser
}

\author{
K. J. Weingarten, D. C. Shannon, and R. W. Wallace \\ Lightwave Electronics, 1161 San Antonio Road, Mountain View, California 94043 \\ U. Keller \\ AT\&T Bell Laboratories, Crawfords Corner Road, Holmdel, New Jersey 07733
}

Received March 28, 1990; accepted June 15, 1990

\begin{abstract}
A diode-pumped Nd:YLF laser has been acousto-optically mode locked at a $2-\mathrm{GHz}$ repetition rate, giving pulse widths of $7 \mathrm{psec}$ with an average power of $135 \mathrm{~mW}$ at a wavelength of $1.047 \mu \mathrm{m}$. The 2-GHz pulse rate corresponds to a free-space cavity length of $7.5 \mathrm{~cm}$, while the laser's actual cavity length is approximately $6.6 \mathrm{~cm}$. The mode locker consists of a sapphire substrate with a lithium niobate transducer, giving $0.5 \%$ loss modulation per watt. Stable mode-locked pulses were achieved for loss modulations of $1 \%$ or greater.
\end{abstract}

Diode-pumped, actively mode-locked solid-state lasers show promise as compact, low-noise, long-lived sources of picosecond pulses. Applications for these lasers include optical testing of integrated circuits (e.g., electro-optic sampling, charge sensing, and voltage-contrast $e$-beam sensing), photonic switching, optical communications, and generation of bursts of picosecond electron pulses for seeding linear accelerators. Desirable qualities for such lasers would be pulse widths of less than 10 psec and relatively large average powers, greater than $100 \mathrm{~mW}$, which would permit power splitting (e.g., to clock a number of detectors) or nonlinear effects (such as frequency conversion or pulse compression). Nd:glass, because of its broad linewidth, has potential for subpicosecond pulses, and several researchers have reported pulse widths of 10 psec or less. ${ }^{1,2}$ However, the low thermal conductivity of Nd:glass leads to thermal lensing and depolarization, limiting its use to low power. Nd:YLF, which has a broader linewidth than Nd:YAG and better thermal properties than glass, has been mode locked with good results by using both amplitude and frequency modulation ${ }^{3-5}$ to frequencies as high as $500 \mathrm{MHz}$. A 1-GHz harmonically modelocked, lamp-pumped Nd:YAG laser was recently demonstrated by using a high-performance GaP mode locker. ${ }^{6}$ We report here a $2-\mathrm{GHz}$ repetition-rate, fundamentally mode-locked Nd:YLF laser producing pulse widths of $7 \mathrm{psec}$ with an average output power of $135 \mathrm{~mW}$ at $1.047 \mu \mathrm{m}$.

As the pulse rate of a mode-locked laser increases, its pulse width should decrease and its stability should increase, owing to a decrease in steady-state buildup time (the number of round trips to achieve mode locking) ${ }^{7}$ and a smaller, more compact, rigid laser cavity. However, the loss modulation of acousto-optic mode lockers decreases at higher drive frequencies owing to increased acoustic attenuation in its substrate and increased losses in the transducer. A recent analysis of acousto-optic mode lockers determined a new figure of merit based on the acoustic (or diffraction) figure of merit $M$, the substrate loss, and the substrate thickness. ${ }^{5}$ This indicated that sapphire, which has a relatively small acoustic figure of merit but excellent low acoustic loss, would perform well as a mode locker at gigahertz drive frequencies. Sapphire also has relatively good optical properties, so that intracavity losses from the device are small and the laser output power remains efficient. The time-varying diffraction with this model is

$$
\eta(t)=\sin ^{2}\left[\frac{\pi}{\sqrt{2} \lambda_{0}} \cos \left(\theta_{B}\right)\left(\frac{M}{\alpha d} \frac{L}{W} P_{0}\right)^{1 / 2} \sin (2 \pi f t)\right],
$$

where $\lambda_{0}$ is the free-space wavelength, $\theta_{B}$ is the Bragg angle, $M$ is the acoustic figure of merit $\left(2.2 \times 10^{-16}\right.$ in SI units for sapphire), $\alpha$ is the acoustic attenuation $\left(2.5 \mathrm{~m}^{-1}\right.$ for sapphire at $\left.1 \mathrm{GHz}\right), d$ is the substrate thickness, $L$ is the transducer length, $W$ is the transducer width, $P_{0}$ is the absorbed drive power, and $f$ is the drive frequency (note that a drive frequency of $f$ results in an optical modulation of $2 f$ ). In practice, the loss modulation is measured, which is the average percentage of light deflected from the main (zero-order) beam owing to diffraction from the acoustic wave. This is approximated by a series expansion of Eq. (1),

$$
\eta_{\mathrm{dc}} \cong \frac{1}{4}\left(\frac{\pi}{\lambda_{0}}\right)^{2} \frac{M}{\alpha d} \frac{L}{W} P_{0}
$$

assuming that the Bragg angle is small and the overall loss modulation is small $(<20 \%)$.

The mode locker was fabricated to the following specifications. The substrate was a 4-mm-thick sapphire étalon, polished parallel to better than 5 arcsec, with its $c$ axis perpendicular to the étalon surfaces. Optical-quality surfaces were polished perpendicular to the acoustic étalon surfaces and antireflection coated for $1.047 \mu \mathrm{m}$. This surface was wedged at $3^{\circ}$ to prevent any étalon effects in the laser cavity. A 


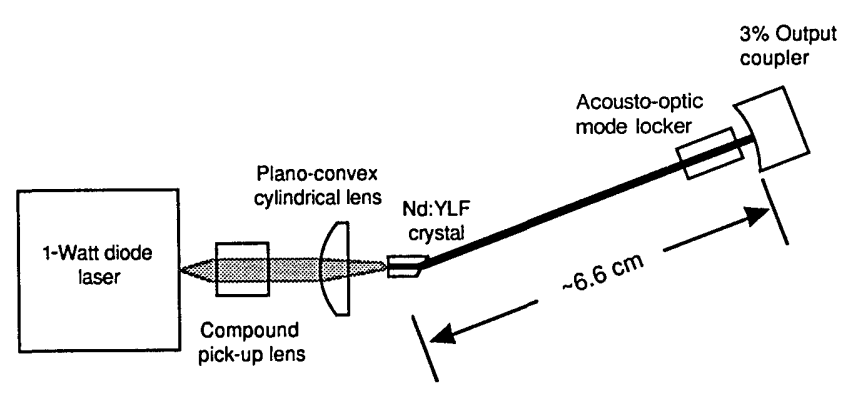

Fig. 1. Schematic of the 2-GHz mode-locked laser cavity.

$\mathrm{LiNbO}_{3}$ transducer, approximately $3 \mu \mathrm{m}$ thick and 0.5 $\mathrm{mm}$ square, was deposited onto the sapphire ${ }^{8}$ so that the acoustic waves were launched along the crystal $c$ axis. The overall device was $4 \mathrm{~mm}$ thick by $6 \mathrm{~mm}$ high by $10 \mathrm{~mm}$ long. The device was wire bonded to a coplanar waveguide/SMA launcher microwave package. For this device, calculations indicated an expected loss modulation of $4.8 \% / \mathrm{W}$ from relation (2).

The experimental setup is as follows. Figure 1 shows the schematic of the laser cavity. The pulse rate of $2 \mathrm{GHz}$ requires a free-space cavity length of 7.5 $\mathrm{cm}$, and the actual cavity length with all the optical elements was approximately $6.6 \mathrm{~cm}$. The Brewsterangled Nd:YLF laser crystal was doped at $1.5 \% \mathrm{Nd}$ concentration, with dimensions of $3 \mathrm{~mm}$ square by 5 $\mathrm{mm}$ long (the nominal length through its middle). The pumped end was flat, coated for high reflection at $1.047 \mu \mathrm{m}(>99 \%)$ and high transmission $(>90 \%)$ at the 798-nm pump wavelength. The Brewster cut provided a low-loss polarizing surface in the laser cavity that ensured that étalon effects would not be present. The laser polarization was parallel to the $c$ axis of the sapphire mode locker. A 1-W pump diode laser (SDL2462) was collected and focused into the laser crystal with a 4-mm focal-length compound spherical lens and a $6.35-\mathrm{mm}$ cylindrical lens to remove the laserdiode astigmatism. The output coupler had a $10-\mathrm{cm}$ radius of curvature and a transmission of $3 \%$. The mode locker was tuned to a resonance at a drive frequency near $1 \mathrm{GHz}$, and the forward power and reflected power were measured with a rf power meter. The signal source was a microwave synthesizer (Hewlett-Packard 8341B) driving a 1-GHz power amplifier capable of up to $6 \mathrm{~W}$ of output power.

The output of the laser was measured with a fast photodiode (Antel Optronics AR-S3) on either a sampling oscilloscope (Tektronix 7704 mainframe with a 7S12, S6, and S-53 sampling unit) or a rf spectrum analyzer (Hewlett-Packard 8591A) and with an optical autocorrelator (background free). The laser supplied enough average power so that, with a suitable beam splitter, both the photodiode and the autocorrelator were monitored simultaneously. The spectrum analyzer was used initially to match the laser free spectral range to the mode-locker modulation frequency, since free-running mode beating could be observed at the laser's free spectral range with the spectrum analyzer. The pulse was then optimized on the sampling scope until it was near the system's response limit, then the autocorrelator was used to measure and optimize the laser performance.

Figure 2 shows a typical autocorrelation (the solid curve) and an ideal hyperbolic secant autocorrelation (the dotted curve) as a comparison. The autocorrelation was taken while the sampling scope was simultaneously monitored, which indicated clean, detectionsystem-limited pulse widths of 60 psec. The best pulse width and shape were obtained with $1 \%$ or better loss modulation, corresponding to $2 \mathrm{~W}$ or greater $\mathrm{ab}$ sorbed power in the mode locker. Note that the mode-locker drive frequency was tunable over several hundred megahertz, in intervals of $1.2 \mathrm{MHz}$ (the resonance spacing due to the 4-mm-thick substrate), permitting the laser repetition rate to be tuned by twice that amount. The data in Fig. 2 were taken for a 2$\mathrm{GHz}$ pulse rate, and mode-locked pulses were obtained with similar pulse widths $(<10 \mathrm{psec})$ for pulse rates down to $1.6 \mathrm{GHz}$. At drive frequencies above 1 $\mathrm{GHz}$ (i.e., pulse rates above $2 \mathrm{GHz}$ ) the amplifier output power rapidly decreased.

The laser stability was good as observed on the sampling scope and the autocorrelator. The pulse width was sensitive to cavity length adjustments, and fine control of the cavity length was accomplished by translating the mode locker in its wedged direction. No special care was taken to isolate the laser from normal room vibrations-the experiment was conducted on a nonisolated workbench, and the laser cavity was open to normal air currents in the room.

The laser had a threshold of approximately $120 \mathrm{~mW}$ of pump power (measured at the laser crystal), a slope efficiency of $28 \%$, and an overall efficiency of $23 \%$, while running mode locked. The output coupling was not optimized, and higher output coupling should give higher average power. The mode-locker insertion loss (with no drive frequency applied) was estimated to be $0.7 \%$.

Additionally, the loss modulation of the mode locker was measured outside the laser cavity. The loss modulation of the device was determined to be $0.5 \% / \mathrm{W}$, compared with the $4.8 \% / \mathrm{W}$ expected from relation (2).

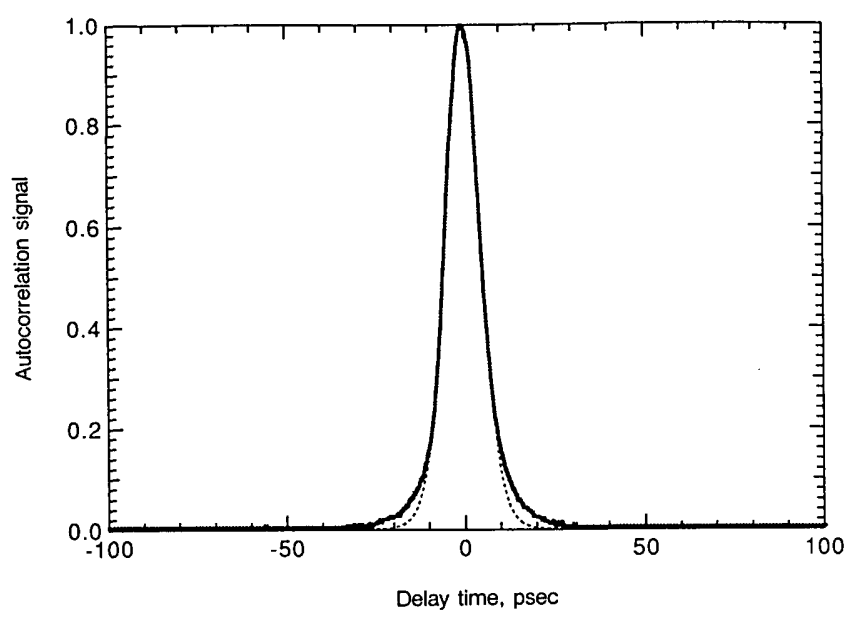

Fig. 2. Measured autocorrelation (solid curve) of the 2$\mathrm{GHz}$ laser with $4.2 \mathrm{~W}$ of absorbed power in the mode locker and the ideal 7.1-psec autocorrelation (dotted curve). 
The model assumes that the losses are dominated by acoustic attenuation. This predicts an upper limit to the loss modulation, because losses due to diffraction of the acoustic wave (causing the acoustic beam to spread out after multiple bounces), loss from substrate wedge (causing the acoustic beam to walk off the transducer after multiple bounces), or loss in the transducer are neglected. The results from Ref. 5 with a $\mathrm{ZnO}$ transducer showed good agreement with the theory, and therefore we believe that loss in the $\mathrm{LiNbO}_{3}$ modulator caused the reduced performance. For the result shown in Fig. 2, the absorbed power of $4.2 \mathrm{~W}$ resulted in a loss modulation of $2.1 \%$. For loss modulations of less than $1 \%$, the laser's autocorrelation began to exhibit wings on the pulse and decreased stability. When the loss modulation was increased to above $1 \%$, the laser pulse width remained stable but the mode locker became easier to align (i.e., the pulse width was less sensitive to transverse positioning of the mode locker) and the pulse width was less sensitive to cavity length changes. The loss modulation could be improved in future mode lockers by decreasing the substrate thickness (which should also decrease loss due to diffraction and the substrate wedge), increasing the transducer length, and decreasing its width. Better loss modulation would mean improved laser performance at lower drive levels, reducing the output power required of the rf amplifier. Additionally, a thin-film transducer, such as $\mathrm{ZnO}$, might also have better performance at gigahertz drive frequencies.

In summary, a 2-GHz repetition-rate mode-locked
Nd:YLF laser giving 7-psec pulse widths has been demonstrated. The laser was pumped with a $1-W$ diode laser giving $135 \mathrm{~mW}$ of average output power at a wavelength of $1.047 \mu \mathrm{m}$. This laser is a compact, stable source of picosecond pulses. Possible applications include testing of optoelectronic components, such as photodiodes, optical generation of picosecond electron pulses, photonic switching, and optical clocking and communications.

This research was partially supported by Small Business Innovative Research Phase I contract DEAC03-89ER80717 from the U.S. Department of Energy.

\section{References}

1. S. Basu and R. L. Byer, Opt. Lett. 13, 458 (1988).

2. L. Yan, P.-T. Ho, C. H. Lee, and G. L. Burdge, IEEE J. Quantum Electron. 25, 2431 (1989).

3. G. T. Maker and A. I. Ferguson, Appl. Phys. Lett. 54, 403 (1989).

4. G. T. Maker and A. I. Ferguson, Electron. Lett. 25, 1025 (1989).

5. U. Keller, K. D. Li, B. T. Khuri-Yakub, D. M. Bloom, K. J. Weingarten, and D. C. Gerstenberger, Opt. Lett. 15, 45 (1990).

6. T. Sizer II, Appl. Phys. Lett. 55, 2694 (1989).

7. A. E. Siegman, Lasers (University Science, Mill Valley, Calif., 1986).

8. Newport Electro-Optics Systems, 4451B Enterprise Court, Melbourne, Fla. 32934. 